\title{
Scopolamine : un nouveau cas de soumission médicamenteuse sur des enfants
}

\author{
Scopolamine used as a drug facilited \\ sedation in children
}

\section{Marie-Laure PUJOL, Marion VILLAIN, Guillaume SALQUÈBRE Emilie VALLET, Vincent CIRIMELE, Pascal KINTZ}

Laboratoire ChemTox, 3 rue Grüninger, 67400 lllkirch, France

*Auteur à qui adresser la correspondance : Dr Pascal KINTZ, Laboratoire ChemTox, 3 rue Grüninger; 67400 Illkirch, France - Tel : 0390400540 - Fax : 0390400541 - e-mail : pascal.kintz@wanadoo.fr

(Article reçu le 16 mai 2006 ; accepté après modifications le 31 juillet 2006)

\section{RÉSUMÉ}

Le but de notre étude était de doser la scopolamine, par UPLC-MS/MS dans les cheveux de 3 enfants d'une même fratrie dans le cadre d'une suspicion de soumission chimique par FEMINAX ${ }^{\circledR}$. Ce médicament, commercialisé en Angleterre, dont les principes actifs sont le paracétamol, la codéine, la caféine et la scopolamine, est indiqué dans le traitement symptomatique des douleurs modérées à fortes (maux de tête, douleurs dentaires, crampes d'estonac, règles douloureuses).

Une mèche de cheveux a été prélevée sur chaque enfant, décontaninée par du dichlorométhane, puis segmentée. Les segments ont ensuite été broyés, puis incubés toute une nuit dans I ml de tampon phosphate pH 8,4 en présence d'un étalon interne $\left(2,5 \mathrm{ng}\right.$ d'atropine-d $\left.d_{3}\right)$. Après extraction liquide/liquide par $5 \mathrm{ml}$ d'un mélange dichlorométhane/isopropanol/ n-heptane $(50 / 17 / 33, \mathrm{v} / \mathrm{v} / \mathrm{v})$, la phase organique a été évaporée à sec. Le résidu a été repris par $100 \mu \mathrm{l}$ de méthanol et $10 \mu L$ sont injectés sur une colonne ACQUITY' C18 $(2,1 \times 50 \mathrm{~mm}, 1,7 \mu \mathrm{m})$ avec un gradient acétonitrilel tampon formate pH 3,O (débit $0,3 \mathrm{ml} / \mathrm{mnin}$ ). La détection est réalisée par un spectromètre de masse triple-quadripôles Quattro Micro ${ }^{\text {Tis }}$ API de Waters Micromass $^{\oplus}$, avec une interface de type électrospray en mode positif (ES+). Chaque composé est identifié par 2 transitions el quantifié

\section{SUMMARY}

The aim of our study was to develop a procedure to test scopolamine by UPLC-MS/MS in the hair of three children after allegation exposure to FEMINAX ${ }^{\boxplus}$.

This pharmaceutical, commercialized in England, contains active ingredients such as paracetamol, codeine, caffeine and scopolamine. This product is proposed in the treatment of headhache, dental pain, related pain and menstrual cramps.

A strand of hair; collected from each child, was decontaninated using dichloromethane, and then segmented. Segments were pulverised and overnight incubated in Iml pH 8.4 phosphate buffer in presence of $2.5 \mathrm{ng}$ atropine- $d_{3}$, used as internal standard. After liquid-liquid extraction with $5 \mathrm{ml}$ dicloromethane/isopropanol/n-heptane $(50 / 17 / 33, v / v / v)$ the organic phase was cvaporated to $d r y-$ ness. The residue was reconstituted in $100 \mu$ l of methanol, from which $10 \mu \mathrm{l}$ were injected into a ACQUITY CIS column $(2.1 \times 50 \mathrm{~mm}, 1.7 \mu \mathrm{m})$ eluted with a gradient of acetonitrile and formate buffer delivered at a flow rate of 0.3 ml/min. A Waters $^{\odot}$ Micromass ${ }^{\oplus}$ Quattro Micro $^{\mathrm{TM}}$ API triple-quadrupole mass spectrometer was used for analysis. Ionization was achieved using electrospray in ES+ mode. Each compound was identified using 2 transitions et quan- 
sur la transition majoritaire ( $\mathrm{m} / \mathrm{z}, 304,06$ à 138,06 pour la scopolamine et 293,12 à 127,06 pour l'étalon interne).

L'analyse segmentaire des cheveux à confirmer la présence de scopolamine dans chaque segment à des concentrations de 0,3 à $1,1 \mathrm{pg} / \mathrm{mg}$.

De plus, des concentrations de codéine, variant de $89 \mathrm{pg} / \mathrm{mg}$ à $544 \mathrm{pg} / \mathrm{mg}$ ont aussi été mises en évidences dans tous les segments, ce qui confirme bien l'exposition. chronique, depuis au moins 4 mois, de ces enfants au médicament FEMINAX ${ }^{\circledR}$.

\section{MOTS-CLÉS}

Scopolamine, cheveux, UPLC-MS/MS, soumission médicamenteuse.

\section{Introduction}

La scopolamine est un alcaloïde présent chez les Solanacées, particulièrement abondant dans les Datura (Datura inoxia, Datura stramonium) et notamment dans les fleurs et les graines. Anger et coll. ont déterminé les concentrations en scopolamine du $D$. inoxia en Nouvelle Calédonie (1). Dans la fleur, la scopolamine était présente à une concentration de $0,34 \mu \mathrm{g} / \mathrm{mg}$, le pistil à $0,38 \mu \mathrm{g} / \mathrm{mg}$ et les graines à $0,22 \mu \mathrm{g} / \mathrm{mg}$. Ces plantes renferment d'autres alcaloïdes comme l'atropine (2), mais dans la plante sèche, la teneur en scopolamine atteint $33 \%$ des alcaloïdes totaux.

De structure chimique très voisine de celle de l'atropine (Figure I) la scopolamine est un antagoniste des récepteurs muscariniques. Chez l'homme, cet alcaloïde exerce une action parasympatholytique responsable d'un syndrome atropinique ou anticholinergique (3) dont les effets sont détaillés dans le tableau I (4).

Tableau I : Signes et symptômes du syndrome anticholinergique.

\begin{tabular}{|cc|}
\hline Niveau périphérique : & Niveau central : \\
\hline Mydriase & Confusion \\
Sècheresse des muqueuses & Agitation \\
Vasodilatation & Hallucinations visuelles et auditives \\
Hyperthermie & Myoclonie \\
Rétention urinaire, constipation & Tremblements et convulsions \\
Tachycardie & Dépression respiratoire \\
\hline
\end{tabular}

L' activité parasympatholytique de la scopolamine est identique à celle de l'atropine, mais moins marquée surtout au niveau myocardique. Néanmoins, il semble que la scopolamine, grâce à son pont époxyde dans le noyau tropane (Figure 1-b), traverse mieux la barrière hémato-encéphalique et est ainsi plus psychoactive. Cliniquement, à faibles doses, la scopolamine est responsable de sédation et d'amnésie ce qui peut en faire une substance de la soumission chimique. De plus, la titated with the major daughter ion $(\mathrm{m} / \mathrm{z} .304 .06$ to 138.06 for scopolamine and 293.12 to 127.06 for the internal standard).

The analysis in each hair segment showed concentrations in scopolamine from 0.3 to $1.1 \mathrm{pg} / \mathrm{mg}$. Furthermore, codeine was identified in each segment, which confirms the children's chronical exposure to FEMINAX ${ }^{\circledast}$.

\section{KEY-WORDS}

scopolamine, Hair; UPLC-MS/MS, drug submission.

scopolamine est une molécule curarisante : elle induit des troubles locomoteurs en inhibant les centres de coordinations motrices ce qui la rend incapacitante (5). A plus fortes doses, le syndrome anticholinergique est majeur avec un tableau de délire atropinique marqué par des hallucinations auditives et visuelles (6).

D'un point de vue thérapeutique, la scopolamine est utilisée en solution injectable d'une part dans le traitement symptomatique des manifestations douloureuses aiguës liées aux troubles fonctionnels du tube digestif et des voies biliaires et des manifestation douloureuses aiguës en gynécologie et dans le traitement en soins palliatifs de l'occlusion intestinale (SCOBUREN ${ }^{\oplus}$ ) et, d'autre part, dans le traitement en soins palliatifs des râles agoniques liés à l'encombrement des voies aériennes supé-

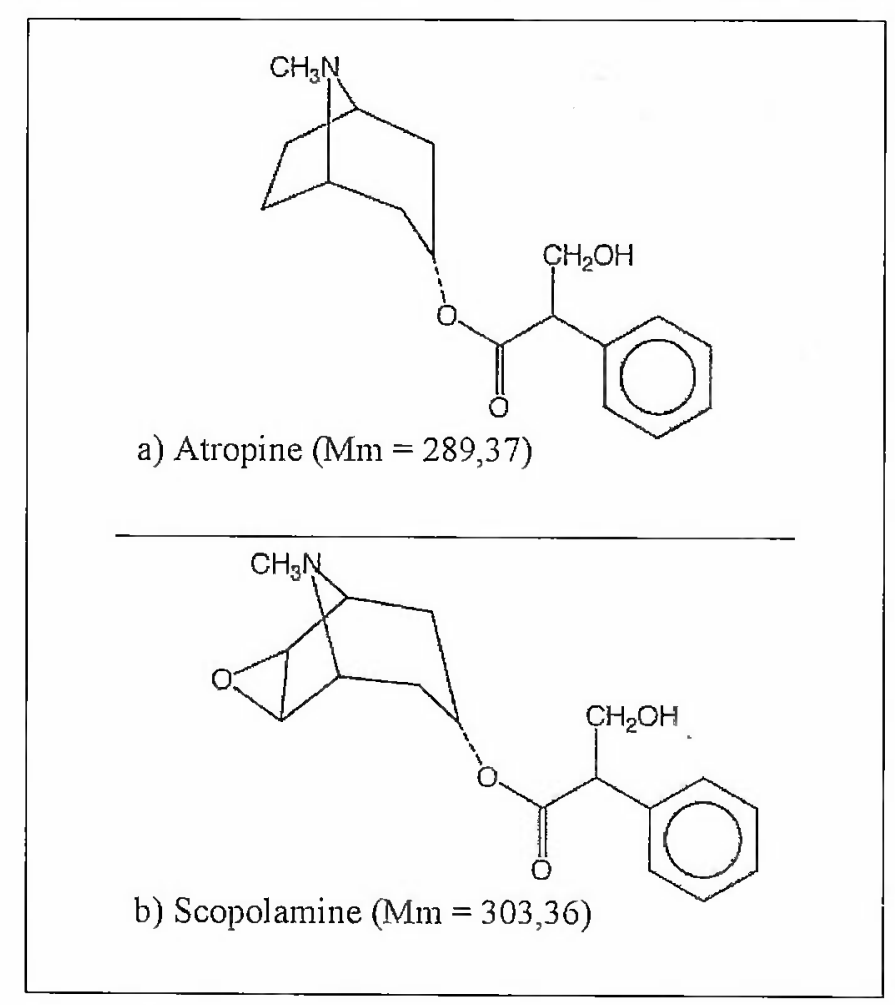

Figure I : Structure chimique de l'atropine (a) et de la scopolamine (b). 
rieures par excès de sécrétions salivaires (SCOPOLAMINE COOPER ${ }^{\oplus}$ ). Enfin, utilisée sous forme de dispositif transdermique, la scopolamine prévient les symptômes du mal des transports (SCOPODERM TTS ${ }^{\oplus}$ ) (7).

Dans cet article, les auteurs rapportent le cas d'une soumission chimique médicamenteuse sur trois jeunes enfants anglais issus de la même fratrie. Le médicament incriminé est le FEMINAX ${ }^{\otimes}$ dont les principes actifs sont le paracétamol, la codéine, la caféine et la scopolamine à des concentrations de $500 \mathrm{mg}, 8 \mathrm{mg}$, $50 \mathrm{mg}$ et $100 \mu \mathrm{g}$ respectivement pour un comprimé. Ce médicament, commercialisé uniquement en Angleterre, est utilisé dans le traitement des douleurs modérées à fortes (maux de tête, douleurs dentaires, crampes d'estomac, règles douloureuses) (8).

La soumission chimique consiste à administrer à l'insu d'une personne, une substance psychoactive à des fins criminelles visant à réduire sa volonté, son libre arbitre, son indépendance ou toute autre forme de pensées d'émancipation physique ou psychique de la victime (9). C'est dans ce cadre que la scopolamine a été testée comme "sérum de vérité » pendant la seconde guerre mondiale. Aujourd'hui, il semble bien que le Datura représente un produit addictif hallucinogène souvent plébiscité par les jeunes et également recherché par des individus peu scrupuleux souhaitant soumettre leurs victimes $(1,10)$.

Une analyse segmentaire des cheveux des 3 victimes a été réalisée par UPLC-MS/MS (chromatographie liquide ultra performance couplé à la spectrométrie de masse en tandem) afin de doser la scopolamine.

En effet, la scopolamine ayant une demi-vie courte (3 à 8 heures) (3), le délai entre l'administration du médicament et les prélèvements biologiques (sang et urines) est souvent trop long pour permettre la détection de la scopolamine. Le cheveux apparaît donc comme une matrice alternative très intéressante.

\section{Matériels et méthodes Solvants et réactifs}

Le dichlorométhane, l'isopropanol, le méthanol ainsi que le n-heptane utilisés sont de qualité HPLC et proviennent de Carlo Erba (Val de Reuil, France). L'acétonitrile, également de qualité HPLC a été acheté chez Merck (Darmstadt, Allemagne).

Pour la réalisation du tampon phosphate ajusté à $\mathrm{pH} \quad 8,4$, le di-ammonium-hydrogénophosphate $\left(\mathrm{NH}_{4}\right)_{2} \mathrm{HPO}_{4}$ - et l'acide chlorhydrique $1 \mathrm{~N}$ nous ont été fournis par Merck (Darmstadt, Allemagne). La solution d'atropine- $\mathrm{d}_{3}$ a été achetée chez Sigma (Saint-Quentin Fallavier, France).

\section{Extraction}

Une mèche d'environ 80 cheveux (diamètre d'un crayon à papier) est prélevée sur chaque enfant en vertex postérieur et orientée au moyen d'une cordelette fixée à $1 \mathrm{~cm}$ de la racine.

Chaque échantillon est décontaminé par 2 bains successifs de dichlorométhane ( $5 \mathrm{ml}, 2 \mathrm{~min})$ puis segmenté ( 3 segments de $2 \mathrm{~cm}$ pour les 2 premiers cas, $2 \mathrm{seg}$ ments de $2,5 \mathrm{~cm}$ pour le troisième). Les segments sont ensuite broyés, puis incubés toute une nuit à $40^{\circ} \mathrm{C}$ dans $1 \mathrm{ml}$ de tampon phosphate ajusté à $\mathrm{pH} 8,4$ en présence de 2,5 ng d'atropine- $d_{3}$ utilisé comme étalon interne. Après extraction liquide / liquide par $5 \mathrm{ml}$ d'un mélange dichlorométhane / isopropanol / n-heptane $(50 / 17 / 33, v / v / v)$, la phase organique est évaporée et le résidu sec repris par $100 \mu$ lde méthanol (11).

\section{Analyse par UPLC-MS/MS}

L'analyse de ces échantillons est réalisée par UPLCMS/MS grâce au système ACQUITY UPLC de Waters. $10 \mu \mathrm{l}$ sont injectés et l'élution est réalisée sur une colonne ACQUITY C18 $(2,1$ x $50 \mathrm{~mm}, 1,7 \mu \mathrm{m})$ avec un gradient acétonitrile / tampon formate pH 3,0 à un débit de $0,3 \mathrm{~m} 1 / \mathrm{min}$ (tableau II).

Tableau II : Gradient de solvants utilisé pour l'analyse de la scopolamine.

\begin{tabular}{|c|c|c|c|}
\hline $\begin{array}{c}\text { Temps } \\
(\mathbf{m i n})\end{array}$ & $\begin{array}{c}\text { Débit } \\
(\mathbf{m l} / \mathbf{m i n})\end{array}$ & $\begin{array}{c}\text { Acétonitrile } \\
(\%)\end{array}$ & $\begin{array}{c}\text { Tampon formate } \\
(\%)\end{array}$ \\
\hline 0 & 0.3 & 5 & 95 \\
\hline 1.30 & 0.3 & 80 & 20 \\
\hline 2.50 & 0,3 & 80 & 20 \\
\hline 3,00 & 0,3 & 5 & 95 \\
\hline 4,80 & 0,3 & 5 & 95 \\
\hline
\end{tabular}

La détection est réalisée par spectrométrie de masse en tandem (QUATTRO MICROTM API, Waters ${ }^{\oplus}$ Micromass $^{\oplus}$ ), avec une interface de type électrospray en mode positif (ES+).

Les conditions suivantes se sont avérées optimales pour l'analyse de la scopolamine et de l'étalon interne : tension de capillaire, $3,0 \mathrm{kV}$; température de la source, $150^{\circ} \mathrm{C}$, température de désolvatation $420^{\circ} \mathrm{C}$, débit de gaz de désolvatation $800 \mathrm{~L} / \mathrm{h}$.

L'acquisition des données est réalisée en mode MRM (Multiple Reaction Monitoring). Les ions parents $[\mathrm{M}+\mathrm{H}]^{+}$sélectionnés et les ions fils détectés ainsi que les paramètres de tension optimaux pour l'analyse sont donnés dans le tableau III. La quantification est réalisée sur les transitions majoritaires $(304,06>138,06$ pour la scopolamine et $293,12>127,06$ pour l'atropine- $\mathrm{d}_{3}$ ). 
Tableau III : Paramètres d'identification de la scopolamine.

\begin{tabular}{|c|c|c|c|c|c|}
\hline Molécules & $\begin{array}{c}\text { Ion parent } \\
(\mathbf{m} / \mathbf{z})\end{array}$ & $\begin{array}{c}\text { Ion fils } \\
(\mathbf{m} / \mathbf{z})\end{array}$ & $\begin{array}{c}\text { Temps de rétention } \\
(\mathbf{m i n})\end{array}$ & $\begin{array}{c}\text { Tension de cône } \\
(\mathbf{V})\end{array}$ & $\begin{array}{c}\text { Energie de collision } \\
(\mathbf{e V})\end{array}$ \\
\hline \multirow{2}{*}{ Scopolamine } & 304 & $\mathbf{1 3 8 ^ { * }}$ & 1,13 & 25 & 23 \\
\cline { 2 - 6 } & 293 & 157 & 1,13 & 25 & 17 \\
\hline Atropine- $\mathrm{d}_{3}$ & $297^{*}$ & 1,20 & 35 & 22 \\
\hline
\end{tabular}

*Ions servant pour la quantification

\section{Résultats}

\section{Validation de la méthode}

La méthode UPLC-MS/MS utilisée dans cette étude est dérivée de notre procédure publiée (11). En particulier, l'analyse par UPLC-MS/MS a permis d'abaisser la limite de détection (LOD) à $0,08 \mathrm{pg} / \mathrm{mg}$ avec une limite de quantification (LOQ) à $0,2 \mathrm{pg} / \mathrm{mg}$. La répétabilité $(\mathrm{n}=6)$ à $50 \mathrm{pg} / \mathrm{mg}$ était de $10 \%$ alors que le coefficient de corrélation de la droite d'étalonnage était de 0.998. La figure 2 représente le chromatogramme obtenu après extraction d'un échantillon de cheveux témoin négatif dopé à une concentration finale en scopolamine de 10 $\mathrm{pg} / \mathrm{mg}$.

\section{Étude du cas}

Les mèches de cheveux ont été prélevées environ 2 mois après la mise en évidence des faits. L'analyse des cheveux montre la présence de scopolamine en quantité faible dans chaque segment. Les résultats sont détaillés dans le tableau IV.

La figure 3 représente le chromatogramme obtenu lors de l'analyse du segment 2,0 à $4,0 \mathrm{~cm}$ du sujet 2 et la figure 4 celui du segment 2,5-5,0 $\mathrm{cm}$ du sujet 3 . Parallèlement, des concentrations de codéine, variant de $89 \mathrm{pg} / \mathrm{mg}$ à $544 \mathrm{pg} / \mathrm{mg}$, ont aussi été mises en évidence les segments de cheveux de ces enfants.

\section{Discussion - Conclusion}

La présence de scopolamine et de codéine dans tous les segments analysés démontrent une exposition chronique de ces 3 jeunes enfants au FEMINAX ${ }^{\oplus}$. Ces 2 molécules ont des propriétés sédatives à concentration thérapeutique. Par conséquent, compte tenu des résultats des ana-

Tableau IV : Résultats de l'analyse segmentaire des cheveux des 3 victimes.

\begin{tabular}{|c|c|c|c|}
\hline \multirow{4}{*}{ Sujet 1 } & $\begin{array}{c}\text { Longueur du } \\
\text { segment (cm) }\end{array}$ & $\begin{array}{c}\text { Masse du } \\
\text { segment (mg) }\end{array}$ & $\begin{array}{c}\text { Concentration èn } \\
\text { scopolamine (pg/mg) }\end{array}$ \\
\cline { 2 - 4 } & $0-2$ & 25 & 0,7 \\
\cline { 2 - 4 } & $2-4$ & 19 & 0,4 \\
\hline \multirow{3}{*}{ Sujet 2 } & $4-6$ & 18 & $<0,2$ \\
\cline { 2 - 4 } & $0-2$ & 31 & 0,6 \\
\cline { 2 - 4 } & $2-4$ & 39 & 0,3 \\
\hline \multirow{3}{*}{ Sujet 3 } & $4-6$ & 38 & $<0,2$ \\
\cline { 2 - 4 } & $0-2,5$ & 32 & 0,3 \\
\hline
\end{tabular}

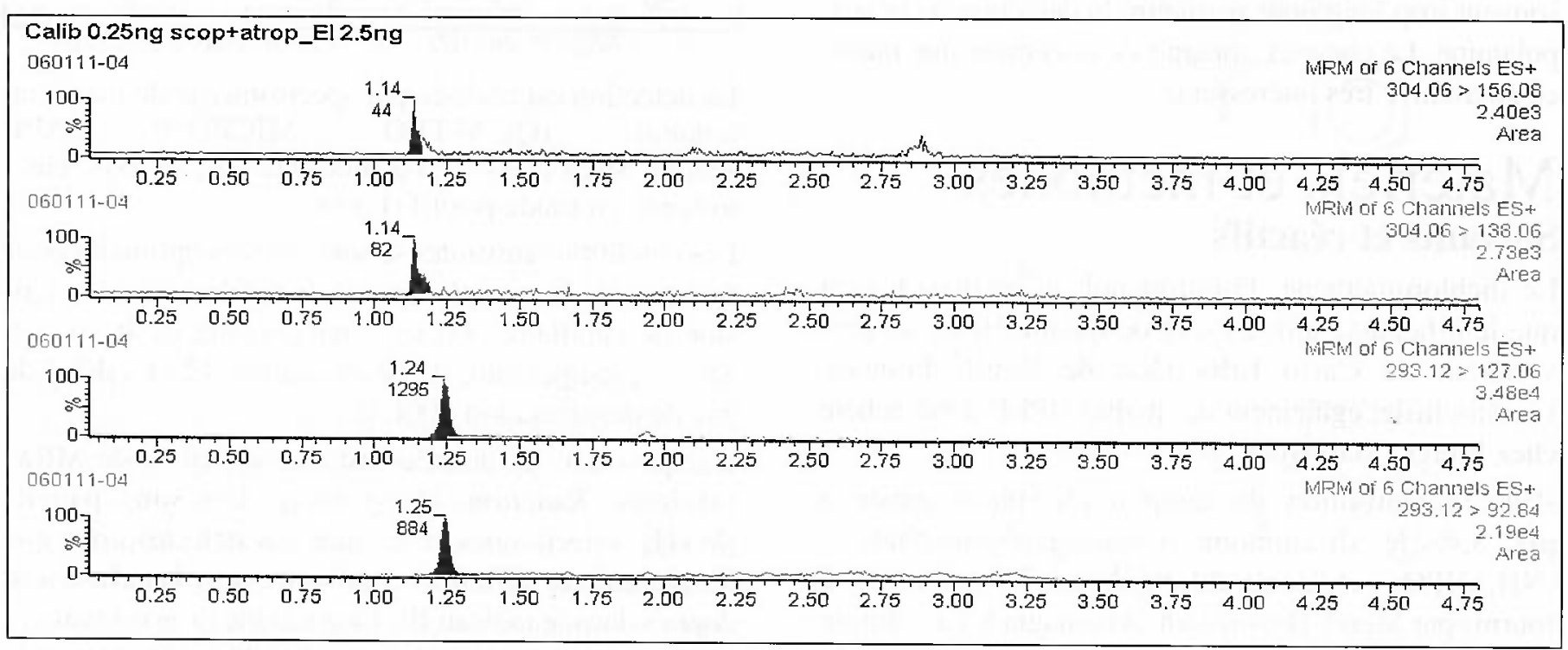

Figure 2 : Chromatogramme d'un échantillon de cheveux dopé à $10 \mathrm{pg} / \mathrm{mg}$ de scopolamine. : En haut: les 2 transitions de la scopolamine, en bas : les 2 transitions de l'atropine- $d_{3}$ 
lyses, il semblerait que le médicament FEMINAX ${ }^{\oplus}$ ait été administré dans le but de sédater ces enfants.

L'analyse des cheveux apparaît ici comme un complément indispensable des analyses conventionnelles sur sang et urines car elle permet d'élargir la fenêtre de détection et de différencier, grâce à la segmentation, une exposition unique d'une exposition chronique.

Alors que la scopolamine devient un sujet préoccupant au vu du nombre de cas, sans cesse croissant, d'intoxications volontaires, en France, aucun cas de soumission chimique par administration de médicament à base de scopolamine n'a été rapporté. Ceci s'explique en autre par le fait que la scopolamine utilisée par voix orale a été retirée du marché depuis une dizaine d'années. Néanmoins, à l'heure d'internet, il est facile de se procurer de tels produits, mais il faut espérer que ce cas ne reste qu'exceptionnel.

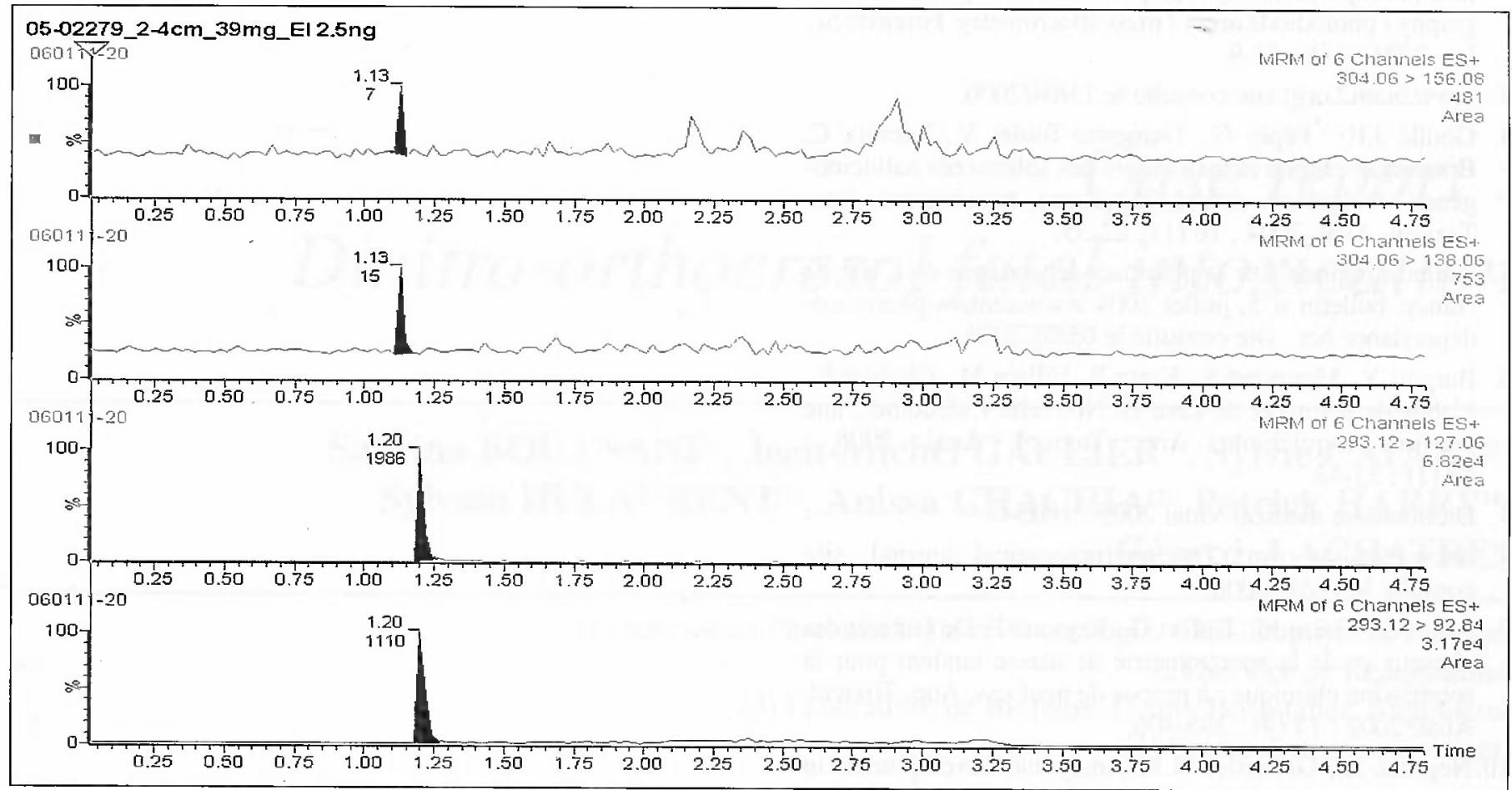

Figure 3 : Chromatogramme d'un échantillon de cheveux prélevé sur le sujet 2 (segment 2-4cni). La concentration en scopolanine est de $0,3 \mathrm{pg} / \mathrm{mg}$. En haut : les 2 transitions de la scopolamine, en bas : les 2 transitions de l'atropine-d;

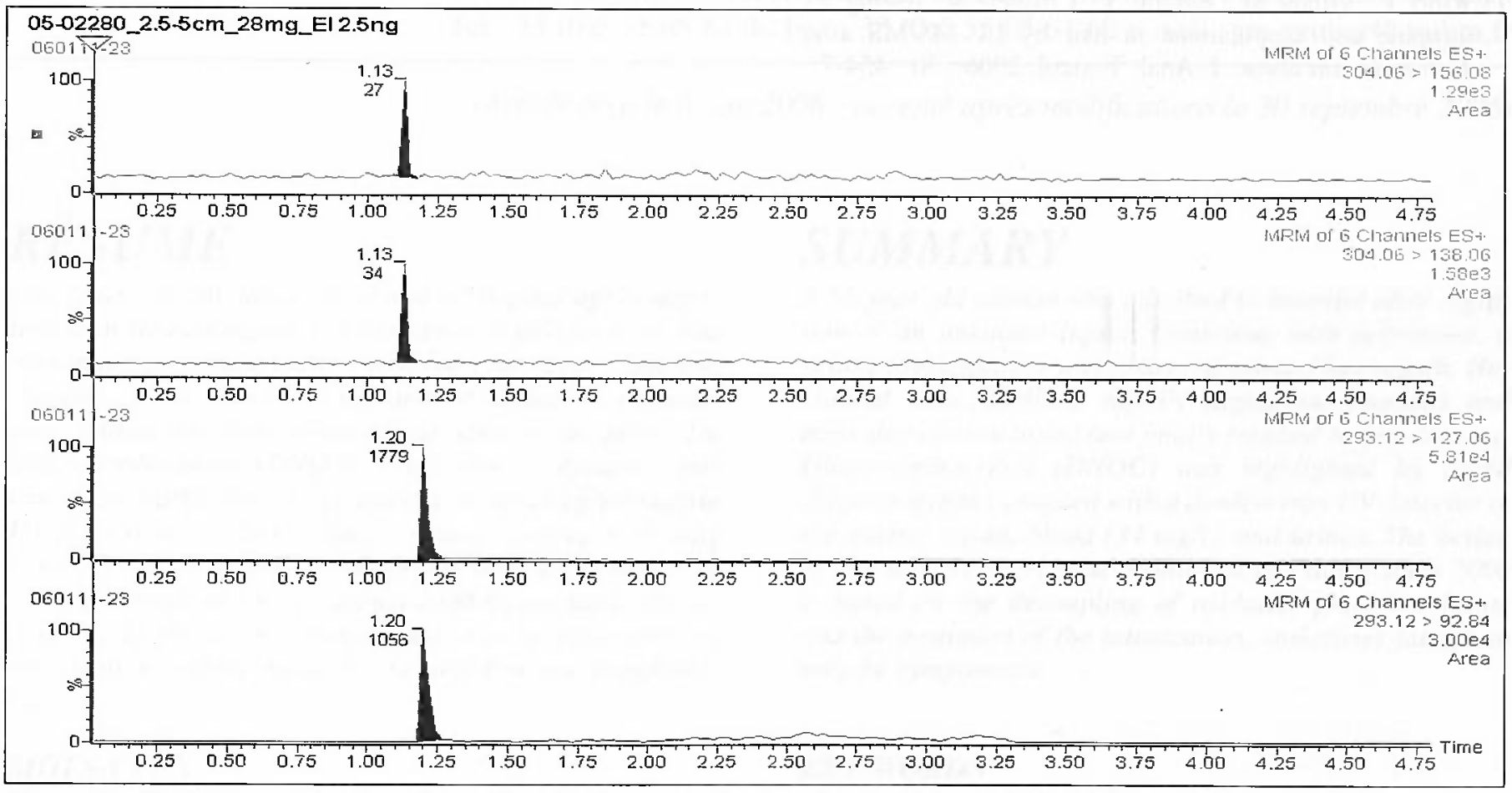

Figure 4 : Chromatogramme d'un échantillon de cheveux prélevé sur le sujet 3 (segment 2,5 à 5,0 cm). La concentration en scopolamine est de 1,1 pg/mg. En haut : les 2 transitions de la scopolamine, en bas : les 2 transitions de l'atropine-d, 


\section{Références}

1. Anger J.P., Villain M., Baert A., Kintz P. Le datura : uneplante abondonnée de la pharmacopée mais qui semble aujourd'hui de plus en plus plébiscitée par les jeunes. J. Med. Lég. Droit Méd. 2005 ; 48 (3) : 185-97.

2. Steenkamp P.A., Harding N.M, van Heerden F.R., van Wyk B.E. Fatal Datura poisoning : identification of atropine and scopolamine by high performance liquid chromatography / photodiode array / mass specrometry. Forensic Sc. Int. $2004 ; 145: 31-9$.

3. www.biam2.org; site consulté le 15/04/2006.

4. Goullé J.P., Pépin G., Dumestre-Toulet V., Lacroix C. Botanique, chimie et toxicologie des solanacées hallucinogènes : belladone, datura, jusquiame, mandragore. Ann. Toxicol. Anal. $2004: 16$ (1) : 22-35.

5. Bulletin régional sur la pharmacodépendance du CHU de Nancy, bulletin $n * 5$, juillet 2004 www.centres-pharmacodependance.net, site consulté le 05/05/2006

6. Barguil Y., Mermond S., Kintz P., Villain M., Choblet E. L'abus de datura et de kava en Nouvelle Calédonie : une pratique inquiétante. Ann. Toxicol. Anal. 2006 ; $18(1): 33-43$.

7. Dictionnaire médical Vidal $2005: 1805-6$.

8. www.Pjonline.com; The pharmaceutical journal, site consulté le 15/04/2006.

9. Pépin G., Chèze M., Dufort G., Vaysette F. De l'intérêt des cheveux et de la spectrométrie de masse tandem pour la soumission chimique : à propos de neuf cas. Ann. Toxicol. Anal. $2002 ; 14$ (4) : 395-406.

10. Negrusz A., Gaensslen R.E. Analytical developments in toxicological investigation of drug-facilitated sexual assault. Anal. Bioanal. Chem. 2003 ; 376 : 1192-7

11.Kintz P., Villain M., Barguil Y., Cirimele V. Testing for atropine and scopolamine in hair by LC-MS/MS after Datura inoxia abuse. J. Anal. Toxicol. 2006 ; 30 : 454-7. 